\title{
THE WAIT-AND-SEE OPTION IN ASCENDING PRICE AUCTIONS
}

\author{
Olivier Compte \\ CERAS-ENPC and CNRS
}

\author{
Philippe Jehiel \\ CERAS-ENPC and \\ University College London
}

\begin{abstract}
Ascending auctions offer agents the option to wait and see before deciding to drop out. We show that in contexts where as time proceeds agents get finer and finer estimates of their valuations, incentives to drop out at one's expected valuation are weak: it is optimal for agents to wait and see. We first illustrate the claim in a private value setting. We next analyze an interdependent value setting in which this wait and see option results in an imperfect information aggregation. We also analyze the implications for the seller's revenue, and show that the ascending format may dominate the second-price format, independently of the date at which the second price auction is run. (JEL: D44, D83)
\end{abstract}

\section{Introduction}

In a private value setup, bidding in an ascending price auction is generally thought of as fairly simple: ${ }^{1}$ A bidder should remain active as long as the price remains below his valuation, and drop out as soon as the price reaches that valuation.

But, the incentives to drop out exactly at one's own valuation are weak, especially if at that price there are still many active bidders. In many instances, it is reasonable to expect bidders to prolong one's participation beyond one's current expected valuation in the hope that some good news will arrive and reveal that the good is worth more.

Specifically, consider a bidder who is somewhat uncertain about how much the object for sale is worth to him, and assume that the price reaches his current expected valuation for the object. What could happen if this bidder were to prolong his participation in the auction beyond his current expected valuation?

\section{E-mail addresses: Compte: compte@enpc.fr; Jehiel: jehiel@enpc.fr}

1. The rules of the ascending price auctions are as follows: the price starts at a low level, and then increases gradually. At any point in time, any bidder can drop out of the auction (and then can no longer come back). The auction stops when there is only one bidder left (or when all remaining bidders drop out simultaneously - in that case the object is allocated to each one of these bidders with equal probability). 
On the one hand, he faces the risk that all remaining bidders drop out of the auction simultaneously. However, if there remains many active bidders this risk seems negligible. On the other hand, he may have second thoughts on the value of the object, or alternatively, he may receive new information inducing him to reassess his valuation for the object. If the new assessment leads to a low valuation, he can always decide to drop out. If the new assessment leads to a high valuation, he may then remain active and enjoy profits with positive probability.

Thus, wait and see seems to be a better option than dropping out. We will check that intuition in a simple private value setting in which one bidder is not certain about his valuation. The setting differs from a standard independent private value setting in one key dimension: The possibility that an agent's valuation be uncertain and changes over time. We shall see that the effect on equilibrium strategies may be significant even when there is only a tiny chance that a bidder's valuation changes. We will next consider an interdependent value setting in which again one agent is uncertain about his signal. We observe that due to the wait-and-see strategy of the latter agent information aggregation is imperfect in the ascending price auction leading to insights that radically differ from the literature.

Finally, we will briefly discuss the effect that these wait-and-see strategies have on revenues, and make concluding remarks.

\section{An Illustration of the Wait-and-See Option: Private Value Setting}

There is one object for sale, worth 0 to the seller. There are $n$ potential buyers, labeled $i=1, \ldots, n$. Buyer $i$ 's valuation is denoted $v_{i}$. Buyers' valuations are assumed to be drawn independently from the uniform distribution with support on $[0,1]$.

Distributions are known to bidders. Initially, at date 0 , all Bidders 2 to $n$ know the realization of their own valuation. Bidder 1 does not. However, there is a chance that he will learn his valuation at some later date. Formally, we denote by $t_{1}$ the date at which Bidder 1 learns his valuation, and we assume that it is distributed according to the exponential distribution with parameter $\mu$, that is,

$$
\operatorname{Pr}\left\{t_{1}>t+\tau \mid t_{1}>t\right\}=\exp \{-\mu \tau\} .
$$

Throughout the paper, we will essentially focus on the ascending price auction. For revenue comparisons, we will also consider the second price auction. In the second price auction, each Bidder $i$ simultaneously submits a bid $b_{i}$. The object is then allocated to the highest bidder at a price equal to the 
second highest bid. ${ }^{2}$ In the ascending price auction, the price starts at 0 and then rises up continuously at constant pace $a .^{3}$ Initially, each player decides whether to be active, and then decides when to drop out of the auction (possibly as a function of the information received during the auction). The price stops rising as soon as there remains only one active bidder. The object is then sold to the remaining bidder at that final price.

The main insight of this section is that ascending formats offer a valuable wait and see option to Bidder 1: Waiting while the current price is already above one's current expected valuation may be costly, because there is a risk of ending up buying the object at a loss, that is, at a price higher than the current expected valuation. However, by waiting, Bidder 1 may obtain additional signals about his valuation, that he may exploit to make a better quitting decision.

Let us analyze formally the incentives to remain active once the current price $p$ lies above Bidder 1's expected valuation $E v_{1}=1 / 2$. Assume that Bidder 1 has not yet learned her valuation and that there remain $m$ active bidders besides Bidder 1. If Bidder 1 remains active a small additional time $\Delta \tau$, he faces the risk that all remaining bidders drop out, in which case he will get a loss of $p-1 / 2$. However, during that lapse of time, he may learn his exact valuation for the object, quit the auction if this realization turns out to be lower than the current price, and remain active otherwise. Let us denote by $G(m, p)$ Bidder 1's expected gain from learning his realization at price $p$ when there remain $m$ other active bidders: ${ }^{4,5}$

$$
G(m, p)=E\left[\max \left(0, v_{1}-\max _{k \in\{2, \ldots, m+1\}} v_{k}\right) \mid \forall k \in\{2, \ldots, m+1\}, v_{k}>p\right] .
$$

Since the price in the auction increases at pace $a$, the event that all other active agents drop out within $\Delta \tau$ has a probability approximately equal to $[a \Delta \tau /(1-p)]^{m}{ }^{6}$. Since $t_{1}$ follows an exponential distribution with parameter $\mu$, the event that Bidder 1 learns his valuation within $\Delta \tau$ has probability $\mu \Delta \tau$. It follows that if

$$
[a \Delta \tau /(1-p)]^{m}(p-1 / 2)<\mu \Delta \tau G(m, p)
$$

Bidder 1 prefers waiting. When $m \geq 2$, this inequality is satisfied for $\Delta \tau$ small

2. In case of ties, each bidder in the pool of highest bidders is selected with the same probability.

3. This model should be viewed as the limit case $(\Delta \searrow 0)$ of a model where the price increases every $\Delta$ by a constant increment $a \Delta$.

4. $G(m, p)$ is strictly positive for all values of $m$ (it is decreasing though with $m$ and converges to 0 as $m$ groes to infinity).

5. Note that because all bidders $2, \ldots, n$ are identical, the identity of the $m$ bidders still active at $p$ is irrelevant.

6. The conditional distribution of the valuation of a bidder still active at $p$ is the uniform distribution on $[p, 1]$, hence the denominator. 
enough, so Bidder 1 always prefers to wait and see. When $m=1$, this inequality will be satisfied if $p$ is not too large. More precisely, let $p^{*}$ solves

$$
(p-(1 / 2)) /(1-p)=(\mu / a) G(1, p) .
$$

Observe that $p^{*}>1 / 2\left(=E\left(v_{1}\right)\right)$. By construction, for any current price $p$ below $p^{*}$, Bidder 1 will stay in, even if there is only one other active bidder. To summarize:

Proposition 1. In equilibrium, so long as she has not learned her exact valuation $v_{1}$, Bidder 1 remains active if there remain at least two other active bidder, or if there remains only one active bidder and the current price is below $p^{*}$.

The economic intuition for the result is as follows. When there are two or more extra active bidders, Bidder 1 takes almost no risk by waiting a little more (the chance that all other remaining bidders drop out simultaneously is negligible relative to the potential gain induced by receiving good news). When there is only one extra active bidder, the expected gains and losses induced by a further short waiting strategy are of comparable size, but when the price is not too far from the expected valuation, the cost is smaller, hence the advantage to the wait and see strategy when the price is not too high (below $p^{*}$ ).

REMARK 1. Note that because Bidder 1 prolongs his participation beyond his current expectation valuation, he may end up making losses ex post, and regret not having dropped out earlier. The equilibrium is thus no longer an ex-post equilibrium.

REMARK 2. Although we have investigated a special structure in which only one bidder is initially uncertain of his valuation, our insight can be shown to apply to settings where several (or even all) may receive information about their valuation during the auction.

REMARK 3. When there is only a tiny chance that Bidder 1 learns his valuation (that is, when $\mu$ is close 0 ), the threshold $p^{*}$ is close to $1 / 2$. The wait and see option is still used in equilibrium (when there are at least two other active bidders), but the effect on the outcome is small. This will not be the case anymore when valuations are interdependent, and we shall see that the effect on the outcome can be large even when $\mu$ is arbitrarily small.

\section{An Interdependent Valuation Setup}

In the private value setup of Section 2, one bidder (Bidder 1) is initially uncertain about his valuation and he may obtain information on his valuation in 
the course of the auction. The interdependent valuation model makes similar assumptions: initially, each bidder only has one signal about his own valuation; In the course of the auction however, through the bidding behavior of their opponents, bidders may learn information about their opponent's signals, hence, because of interdependence, on their own valuation as well. Despite the resemblance with the example studied above, bidders do not seem to be using a wait and see strategy. The purpose of this section is to clarify why.

Consider the following setting. There is one object for sale, worth 0 to the seller. There are three potential buyers, labeled $i=1,2,3$. Bidder $i$ 's valuation is denoted $v_{i}$. Each bidder receives a signal $\theta_{i}$. Valuations are assumed to satisfy:

$$
\begin{aligned}
& v_{1}=\theta_{1}+\alpha \theta_{2}, \quad \text { with } 0<\alpha<1 \\
& v_{2}=\theta_{2} \\
& v_{3}=\theta_{3} .
\end{aligned}
$$

The signals $\theta_{i}$ are assumed to be drawn independently from uniform distributions with support on $[0,1]$.

Equilibrium behavior in the ascending auction is well known. For Bidders 2 and 3 , it is a dominant strategy to remain active up to their respective valuation. For Bidder 1, consider the event where Bidder 2 has not dropped out. ${ }^{7}$ In that event, it is optimal for Bidder 1 to remain active as long as the price $p$ does not exceed his expected value, conditional on the event where Bidder 2 would drop out at $p$. That is, he remains active as long as

$$
p<E\left[v_{1} \mid \theta_{1}, 2 \text { drops out at } p\right]
$$

or equivalently,

$$
p<\theta_{1}+\alpha p .
$$

Thus when the price reaches $p^{*}\left(\theta_{1}\right)=\theta_{1} /(1-\alpha)$ and Bidder 2 has not yet dropped out, Bidder 1 is in a situation that looks quite similar to that of the previous example: he is uncertain about the valuation of the object, because he does not know Bidder 2's signal; he could learn more about his valuation by waiting more. Yet he drops out. Why can't we extend the intuition of the previous example to this case?

The reason is as follows. Once the current price reaches $p^{*}\left(\theta_{1}\right)$, he knows that $\theta_{2} \geq p^{*}\left(\theta_{1}\right)$, hence whatever additional information Bidder 1 learns about $\theta_{2}$, Bidder 1 cannot expect to make any gain: he will pay at least $\theta_{2}$ for the object, so he cannot expect to gain more than

7. In the event where Bidder 2 has dropped out, the price at which Bidder 2 dropped out, say $p_{2}$, reveals Bidder 2's signal, and it is then optimal for Bidder 1 to remain active up to price $\theta_{1}+\alpha p_{2}$. 


$$
\theta_{1}+\alpha \theta_{2}-\theta_{2}
$$

which is smaller than

$$
\theta_{1}+\alpha p^{*}\left(\theta_{1}\right)-p^{*}\left(\theta_{1}\right)=0
$$

when $\theta_{2}>p^{*}\left(\theta_{1}\right)$.

So Bidder 1 cannot expect any gain from waiting beyond the price $p^{*}\left(\theta_{1}\right)$. This example sheds light on one important feature of our previous example: even if the chance of getting new information is very small, it is important that the new information generates (with positive probability) a significant change in the bidder's estimation.

\section{Information Aggregation in Ascending Auctions}

One virtue of the ascending auction is that bidding behavior conveys each bidder some information about other bidders' signals. This is useful in interdependent value contexts, because it promotes efficiency. In the interdependent value setting of Section 2, for example, the equilibrium allocation is efficient in the ascending auction, whereas it would not be efficient in the second price auction (see Krishna (2003) for an analysis building on Dasgupta and Maskin (2000) of when ascending price auctions induce efficient outcomes in onedimensional interdependent value one-object setups).

Whether information is transmitted in equilibrium critically depends on bidders' equilibrium behavior. In Section 3, Bidder 1 obtains precise information about Bidder 2's signal in equilibrium, because Bidder 2's drop-out price perfectly signals Bidder 2's valuation. The analysis of Section 2 however suggests that bidding behavior may be quite sensitive to prospects for information acquisition, even if these prospects are rather unlikely. And as a consequence, information aggregation may be seriously impaired when such prospects exist.

For the sake of illustration, modify our interdependent value example of Section 3 by assuming that valuations satisfy:

$$
\begin{aligned}
& v_{1}=\theta_{1}+\alpha \theta_{2}, \quad \text { with } 0<\alpha<1 \\
& v_{2}=\theta_{2}+\varphi \\
& v_{3}=\theta_{3} .
\end{aligned}
$$

So the only difference with the example of Section 3 is that Bidder 2's valuation is now the sum of two terms, $\theta_{2}$ and $\varphi$. As before, we assume that the signals are drawn independently from uniform distributions on $[0,1]$. We also assume that $\varphi$ is drawn independently from a uniform distribution on $[-1,1]$. 
Finally, we assume that initially, each bidder $i$ only knows the realization $\theta_{i}$, and that over time Bidder 2 may learn the realization $\varphi$. As in Section 2, the date at which learning occurs is assumed to follow an exponential distribution with parameter $\mu$. In the case where $\mu=0$, no learning occurs, and the analysis would be identical to that of the previous section.

We are interested in the limit case where $\mu$ is positive but arbitrarily small. In this limit case, the behavior of Bidder 2 follows much the same logic as that developed in Section 2. That is, Bidder 2 will stay in as long as the other two bidders are still active, ${ }^{8}$ and when there is only one other active bidder, Bidder 2 will drop out if her expected valuation $\left(\theta_{2}\right.$ almost surely) is below the current price. This in turn allows us to derive:

Proposition 2. The following behavioral strategies constitute the limit of an equilibrium as $\mu$ converges to 0 . Bidder 3 remains active until the price reaches his valuation, and drops out at that price. Bidder 2 waits until there is only one other active bidder left. Once this is the case, he drops out if and only if the current price is no smaller than his expected valuation $\theta_{2}$. If Bidder 3 has dropped out and Bidder 2 is still active, Bidder 1 remains active until $p^{*}\left(\theta_{1}\right)=$ $\theta_{1} / 1-\alpha$. If neither player has dropped out, Bidder 1 remains active until price $p^{* *}\left(\theta_{1}\right)$ which solves:

$$
F(p) E\left[v_{1}-p \mid \theta_{2}<p\right]+\int_{p}^{p^{*}\left(\theta_{1}\right)}\left(v_{1}-\theta_{2}\right) f\left(\theta_{2}\right) d \theta_{2}=0 .
$$

Observe that $p^{* *}\left(\theta_{1}\right)<p^{*}\left(\theta_{1}\right)$, and recall that $p^{*}\left(\theta_{1}\right)$ corresponds in the analysis of Section 3 to the price at which Bidder 1 drops out when Bidder 2 is still active.

To get an intuition for Proposition 2, note that compared to Section 3, Bidder 2's behavior differs in the essential feature that she no longer drops out unconditionally at price $\theta_{2}$, but she drops out only when there is one other active bidder and the price is not below $\theta_{2}$ (see the discussion preceding the Proposition). This in turn makes it harder for Bidder 1 to infer Bidder 2's signal from her quitting decision. Specifically, when at price $p$ Bidder 3 quits first and is then followed by Bidder 2, Bidder 1 can only infer that $\theta_{2}$ is smaller than $p$, not the exact value of $\theta_{2}$. Assume now that all three bidders are active and consider Bidder 1's decision whether to stay till $p+d p$ or quit at $p$. The only difference between the two quitting decisions is when Bidder 3 quits in the interval $(p, p+$ $d p$ ). Then either Bidder 2's signal $\theta_{2}$ is below $p$ in which case Bidder 2 quits as well resulting for Bidder 1 in an expected payoff of $F(p) E\left[v_{1}-p \mid \theta_{2}<p\right]$ or

8. For this it is important that the upper bound of the support of $\varphi$ be sufficiently large so that learning good news on $\varphi$ result in a profit at whatever price. 
Bidder 2's signal $\theta_{2}$ is above $p+d p$ resulting for Bidder 1 in an expected payoff of $\int_{p}^{p^{*}\left(\theta_{1}\right)}\left(v_{1}-\theta_{2}\right) f\left(\theta_{2}\right) d \theta_{2}$. The formula (1) follows.

As should be clear from the above discussion, information transmission is no longer perfect in equilibrium, and the allocation is not necessarily efficient. For example, consider the event where

$$
p^{* *}\left(\theta_{1}\right)<\theta_{3}<\theta_{2}<p^{*}\left(\theta_{1}\right)
$$

Under this event, efficiency would require to allocate the object to Bidder 1 (because $\theta_{2}<p^{*}\left(\theta_{1}\right)$ implies $\left.\theta_{2}<\theta_{1}+\alpha \theta_{2}\right)$. However because $p^{* *}\left(\theta_{1}\right)<$ $\min \left(\theta_{2}, \theta_{3}\right)$, Bidder 1 drops out first, and eventually Bidder 2 obtains the object. It is the fear that Bidder 2 stays in only in the hope of learning good news (i.e., that $\varphi$ is high) that eventually leads Bidder 1 to quit first without learning Bidder 2's signal.

\section{Implications for Revenues}

Here we discuss the effect of using an ascending auction rather than a second price auction, in a setting similar to that analyzed in Section 2. We consider an independent private value setting where bidders may get more precise estimates of their valuation over time.

A first issue is: How do changes in the precision of bidders' information affect the seller's revenue in a second price auction. As we have already shown elsewhere (Compte and Jehiel 2000), the effect is ambiguous, and in general depends on whether competition is fierce or not. ${ }^{9}$ With only two bidders competing, for example, the effect cannot be positive because for any realization of $\theta_{2}, E \min \left(\theta_{1}, \theta_{2}\right) \leq \min \left(E \theta_{1}, \theta_{2}\right)$, hence expected revenue is smaller when bidder knows $\theta_{1}$ than when he does not.

To see why the effect is ambiguous with more than two bidders, consider the case of $n$ bidders, $(n-1)$ of whom say $2, \ldots, n$ have a precise value for their valuation, and let $\theta^{(1)}$ and $\theta^{(2)}$ denote respectively the largest and second largest valuations among these $(n-1)$ bidders. If $E \theta_{1}>\theta^{(1)}$, then learning by Bidder 1 may only decrease revenues below $\theta^{(1)}$. In contrast, if $E \theta_{1}<\theta^{(2)}$, then learning by Bidder 1 may only raise revenues above $\theta^{(2)}$.

On average however, when competition is fierce enough, the second event becomes more likely and the effect of precision on revenues turns out to be positive.

9. Note that the insight of Milgrom and Weber (1982) does not apply here. They show that revenues do not decrease when bidders receive additional information that is affiliated with their own signal. Here, learning the actual realization is tantamount to receiving additional information. However, Milgrom and Weber's result requires that new information affect all bidders' valuation in identical ways, an assumption that is not made here. 
In the ascending price auction, bidder's information get more precise over time. So running an ascending auction instead of a second price auction has a mechanical effect on revenues, which we identified above. In addition to this mechanical effect, it is easy to see that in the ascending auction, a bidder's expected drop out price is larger than his second price auction bid.

Indeed, in the event where Bidder 1 does not learn his realization, he is ready to wait till the price reaches some $p^{*}>1 / 2$ before he drops out. And in the event where Bidder 1 learns his realization at price $p$, his expected drop out price is $E \max \left(p, v_{1}\right)$, which is larger than $E v_{1}$.

A consequence of this increase in drop-out prices is an increase in revenues for the seller. In particular, consider the private value setting of Section 2 with $n=3$. One can show that the ascending price auction generates more revenue than the sealed-bid second price auction irrespective of the date at which the sealed bid auction is performed. The revenues are higher in the ascending price auction essentially for two reasons. Because Bidder 1 remains active beyond his expected valuation for the object, he may win the object at a loss. Even when he does not win the object, and say Bidder 3 wins the object, expected revenue for the seller increases because Bidder 1 remains active longer.

\section{Concluding Remarks}

This paper is related to our earlier work on information acquisition in auctions (Compte and Jehiel 2000). In the latter paper, bidders may decide to acquire information, at some cost $c$ (at any point in time during the auction). We found out that it could be worthwhile for bidders to remain active above their current expected valuation, and decide later whether to acquire information. This is typically the case when there are at least three bidders active and one of them may acquire information: There is then essentially no risk to remaining active, as one can always wait until the date where only one other bidder remains active. When there remain only two active bidders however, our conclusion was that bidders should not wait further above their current valuation and either drop out or immediately acquire information. This is in contrast with the current paper, which found a positive value to waiting even when there are only two active bidders.

The reason is as follows. In the current paper, although information arrives freely, one has to remain active to be able to use it. So, there is an implicit cost to information acquisition, in the form of prolonged participation in the auction. In contrast with our previous work however, this cost is endogenous and depends on the current price. When the price is close to the expected valuation, this cost is very small, and is always worth the expected benefit. 
Also, both works find an advantage to the ascending format, yet the reasons differ. In our earlier work, revenues to the seller increase when he runs an ascending auction because in this format, bidders acquire information more often. So revenue increases stem from changes in the information structure. What this paper provides is an alternative reason as to why the ascending auction generates higher revenues, which is not necessarily based on changes in the information structure. Revenues increase in the ascending format because delayed learning increases the price paid by winners. Revenues also increase because bidders are ready to pay for information, and indeed do pay for information, in the form of prolonged participation. In contrast to our earlier paper however, this "payment" is not wasted: a bidder may end up buying the object at a price higher than its current expected valuation; and even if he does not, this raises even further the price paid by winners.

Finally, ${ }^{10}$ building on Compte and Jehiel (2000), Rasmussen (2001) considers a two-bidder dynamic auction with deadline inspired from internet auctions in which one bidder can refine his valuation at a cost. He observes that such a setup may explain that the informed bidder may wait till close to the deadline (before submitting) his bid in an attempt to avoid the uninformed bidder to examine his value.

\section{References}

Compte, Olivier and Philippe Jehiel (2000). "On the Virtues of the Ascending Price Auctions: New Insights in the Private Value Setting." Working paper, CERAS and UCL.

Dasgupta, Partha and Eric Maskin (2000). "Efficient Auctions." Quarterly Journal of Economics, 115, 341-388.

Krishna, Vijay (2003). "Asymmetric English Auctions.” Journal of Economic Theory, 112, 261-288.

Milgrom, Paul and Robert Weber (1982). "A General Theory of Auctions and Competitive Bidding." Econometrica, 50, 1089-1122.

Parkes, David (2003). "Auction Design with Costly Preference Elicitation.” Working paper, Harvard University.

Rasmussen, Eric (2001). "Strategic Implications of Uncertainty over One's Own Private Value in Auctions." Working paper, Indiana University.

Sandholm, Tuomas W. (2000). "Issues in Computational Vickrey Auctions." International Journal of Electronic Commerce, 4, 107-129.

10. There are also a few computer science authors who have considered setups where bidders are initially uncertain about their own valuation. For example, Sandholm (2000) observes in this setup that Vickrey-Clarke-Groves mechanisms lose their property of dominant strategy implementation (because, for example, the decision to refine your valuation may be affected by how close you think other valuations are). Parkes (2003) also considers various auctions formats in which bidders try to elicit their valuations. 